Abstract IDDF2019-ABS-0075 Table 1 Differentially expressed metabolites between $\mathrm{pc}$ and $\mathrm{dm}$

\begin{tabular}{llllll}
\hline name & ttest & $\begin{array}{l}\text { fold } \\
\text { Change } \\
\text { (PC/DM) }\end{array}$ & VIP & mass & Mode \\
& & & & \\
\hline C16 & 0.000 & 0.57 & 2.192 & 351.2167 & Neg \\
Sphingosine-1-phosphate & & & & & \\
keto palmitic acid & 0.000 & 6.15 & 2.536 & 270.2185 & Neg \\
Isoleucine & 0.000 & 0.76 & 2.260 & 131.0945 & Neg \\
N-Succinyl-L- & 0.000 & 0.19 & 2.880 & 290.1123 & Neg \\
diaminopimelic acid & & & & & \\
Uridine & 0.000 & 0.67 & 2.056 & 244.0692 & Neg \\
3-ketosphingosine & 0.000 & 4.10 & 2.500 & 297.2674 & Pos \\
Arachidonoyl dopamine & 0.000 & 0.48 & 2.319 & 439.3074 & Pos \\
PE(18:2) & 0.000 & 0.58 & 2.594 & 477.2868 & Pos \\
Ubiquinone-1 & 0.000 & 0.38 & 2.939 & 250.121 & Pos \\
Valine & 0.007 & 0.81 & 2.573 & 117.0792 & Pos \\
\hline
\end{tabular}

metabolomics to discriminate pancreatic cancer (PC) from new onset diabetic mellitus.

Methods Serum samples from patients with new-onset diabetic mellitus ( $\mathrm{n}=30)$ and new onset diabetic mellitus with PC $(\mathrm{N}=30)$ were examined by liquid chromatography-mass spectrometry (LC-MS). Data were analyzed using orthogonal projection to latent structures (OPLS) and t-test for the differentially expressed metabolites between two groups. The diagnostic model was established using Logistic regression via a stepwise method on the first 15 new onset diabetic mellitus and new onset diabetic mellitus with PC training set. It was then evaluated in the late 15 new onset diabetic mellitus and new onset diabetic mellitus with PC testing set.

Results Five metabolites identified in positive ion model were 3-ketosphingosine, Arachidonoyl dopamine, PE(18:2), Ubiquinone-1 and Valine. C16 Sphingosine-1-phosphate, keto palmitic acid, Isoleucine, N-Succinyl-L-diaminopimelic acid and Uridine in negative ion model were identified to be differentially expressed in the two groups. The regression model established in training set was $\mathrm{p}=\mathrm{e}(\mathrm{X} \beta) /(1+\mathrm{e}(\mathrm{X} \beta)),(\mathrm{X} \beta)=$ $158.975-1.891$ (Age) +0.309 (PE18:2) + 1.035 (C16_Sphingosine_1_phosphate) + 0.084 (Isoleucine) + 1.1145 ( N_Succinyl_L_diaminopimelic_acid). The AUC of this model was 0.982 in testing set; the sensitivity and specificity were both $93.3 \%$. (table 1).

Conclusions Serum metabolomics-based diagnostic approach is a promising method for screening PC from new onset diabetic mellitus.

\section{IDDF2019-ABS-0083 THE USE OF HEMOSPRAY ENDOTHERAPY IN PATIENTS WITH ACUTE GASTROINTESTINAL BLEEDING: A PHILIPPINE EXPERIENCE}

Enrik John Aguila*, Juliet Cervantes, Joseph Erwin Dumagpi. St. Luke's Medical Center Global City, Philippines

\subsection{6/gutjnl-2019-IDDFabstracts. 138}

Background Hemospray (TC-325) is a novel hemostatic agent recently licensed for endoscopic hemostasis of non-variceal upper gastrointestinal (GI) bleeding in Europe, Canada and America. It acts by creating a mechanical barrier and effecting hemostasis on actively bleeding lesions via endoscopy. After an extensive search of publications, the group only found one research describing the experience of hemospray use in the local Asian setting done in 2011. This study aims to describe the effectiveness of hemospray therapy on patients treated at the St. Luke's Medical Center, Global City (SLMC GC), Philippines.

Methods A retrospective study was done on consecutive adults with upper and lower active gastrointestinal tract bleeding treated with hemospray at SLMC GC since its introduction in 2017. Data on patient profile, details of the procedure, and indications of hemospray use either as monotherapy or as salvage therapy were collected. Outcomes including primary hemostasis and re-bleeding rates were analyzed.

Results From 2017 to 2018, a total of 8 patients with acute GI bleeding were managed with hemospray at SLMC GC. With an age range of 36-83 years old, these patients presented with hematochezia, hematemesis or melena. $\underline{3}$ of the cases had a bleeding gastric mass, 3 had bleeding ulcers, while 2 had portal hypertensive bleeding. Hemospray powder was applied on the identified bleeding sites via endoscopy (figure 1A)(figure 1B.). The rate of successful initial hemostasis after hemospray was $87.5 \%$ (7/8). In terms of the endoscopic hemostatic methods used, of the 8 patients, 4 (50\%) had
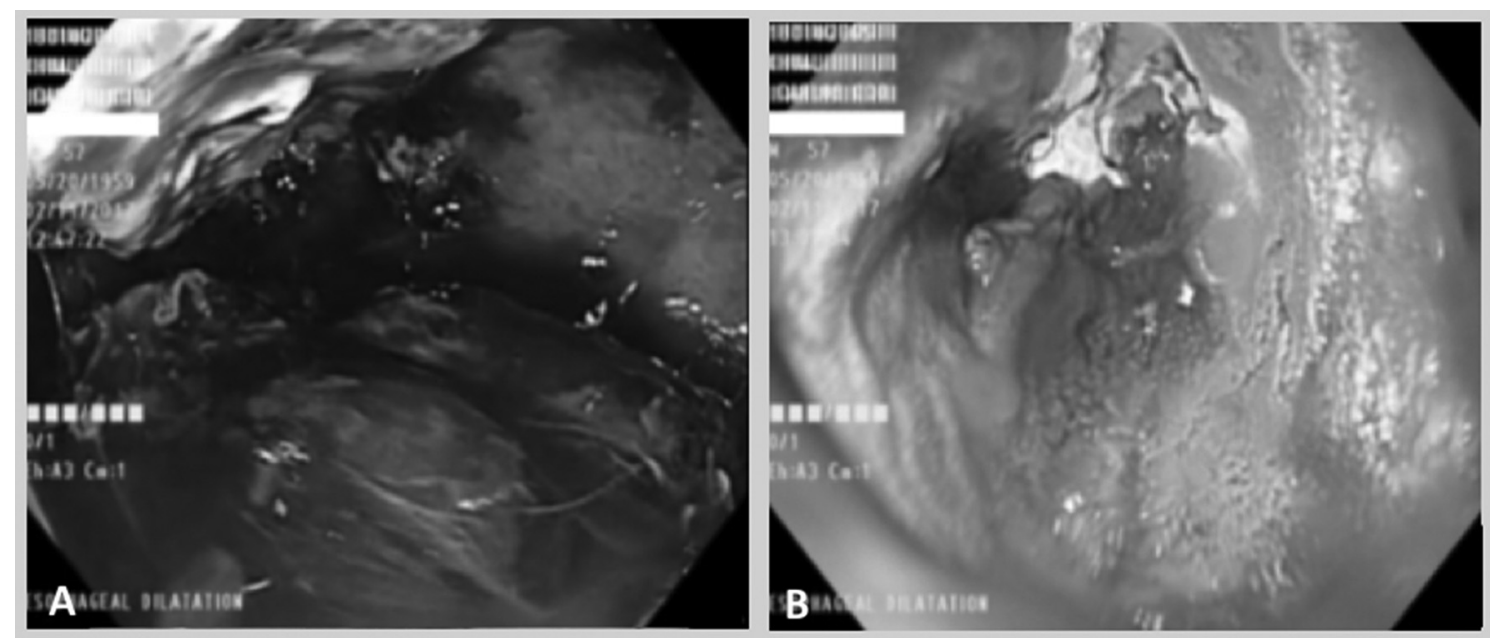

Abstract IDDF2019-ABS-0083 Figure 1 A) Ulcerating gastric mass at the corpus; B) Successful hemostasis after hemospray application 


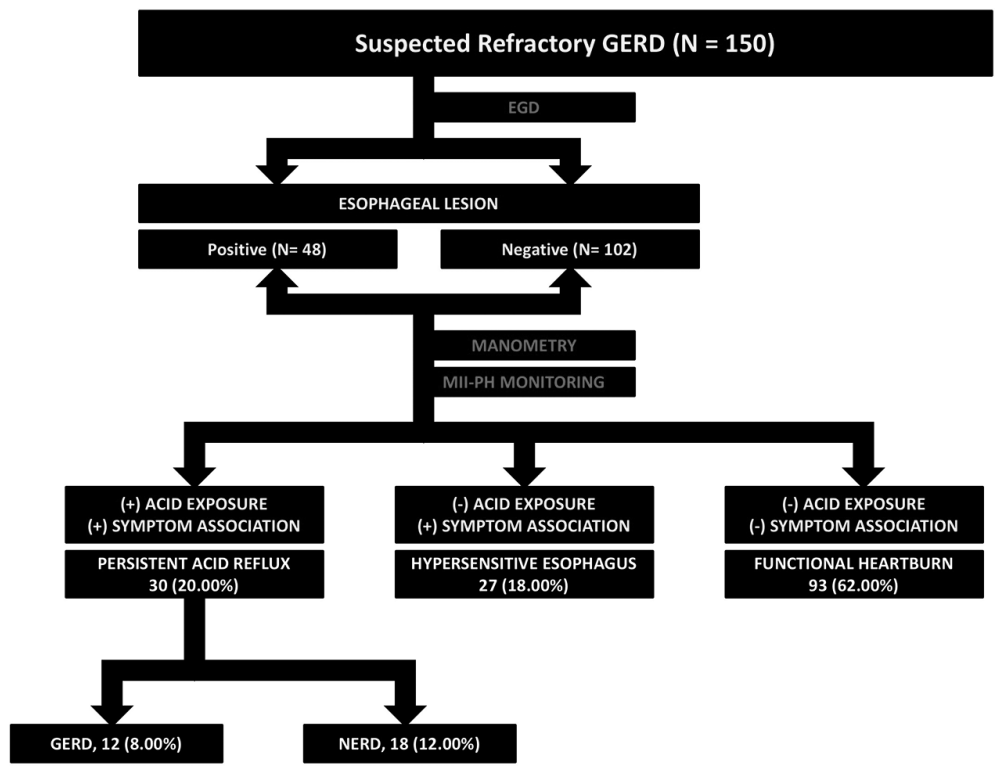

Abstract IDDF2019-ABS-0085 Figure 1 Proportion of patients according to phenotypic group

hemospray as monotherapy and another $4(50 \%)$ as salvage therapy after argon plasma coagulation, endoclipping or epinephrine sclerotherapy did not achieve hemostasis. All patients $(100 \%)$ achieved immediate hemostasis. Only 1 (12.5\%) patient rebled within the 7 days post-hemospray application for which he underwent surgery 5 days after hemospray due to persistent hematochezia.

Conclusions Hemospray is a novel endoscopic technique that has advantages of being non-traumatic, noncontact and can cover large areas of mucosa. This report supports existing studies and demonstrates that hemospray is a safe and effective endoscopic therapy in achieving initial hemostasis, both as primary and salvage therapy in different etiologies of bleeding.

\section{IDDF2019-ABS-0085 THE YIELD OF COMBINED MULTICHANNEL INTRALUMINAL IMPEDANCE AND PH MONITORING (MII-PH MONITORING) AMONG PATIENTS WITH SUSPECTED REFRACTORY GASTROESOPHAGEAL REFLUX DISEASE: A SIX-YEAR EXPERIENCE IN A MULTICENTER, TERTIARY LEVEL HOSPITALS IN THE PHILIPPINES}

Marc Julius Navarro*. St. Luke's Medical Center, Quezon City, Philippines

\subsection{6/gutjnl-2019-IDDFabstracts. 139}

Background Multichannel intraluminal impedance used in combination with pH-metry (MII-pH) has been shown to allow accurate recording of gastroesophageal reflux at all $\mathrm{pH}$ levels. In the Philippines, there is no local data on the yield of these tests in the investigation of the patients with suspected refractory GERD as well as on the phenotypic profiles of Filipino patients being referred for esophageal reflux monitoring. The main objective of the study is to determine the yield of combined multichannel intraluminal impedance and $\mathrm{pH}$ monitoring among patients with suspected refractory GERD at St. Luke's Medical Center, Quezon City and Global City.

Study design Retrospective, Cross-sectional, Chart Review.

Participants Suspected Refractory GERD patients who underwent MII-pH.

Statistical analysis Descriptive statistics.

Results There is a total of 150 subjects included in the study. Majority of the patients was in their 4th to 5 th decade of life, presented with typical symptoms of reflux, specifically heartburn $(44.00 \%)$ and regurgitation $(40.00 \%)$. In terms of EGD findings, $52.00 \%$ of patients have no signs of esophagitis. The manometric findings most commonly revealed normal esophageal motor function (52.00\%). It can be noted that patients with phenotypic group of persistent acid reflux, revealed an overall prevalence of $20.00 \%$. Around $40.00 \%$ of patients presented with esophagitis on EGD, alongside with normal manometric findings (60\%), and positive SI $(60.00 \%) /$ positive SAP (100\%). Whereas those patients under the phenotypic group of hypersensitive esophagus, revealed an overall prevalence of $18.00 \%$. Patients mostly presented without esophagitis on EGD (77.78\%), 55.56\% with normal esophageal motor function on manometry, and all patients have positive SAP (100\%)/ negative SI (100\%). On the other hand, in patients labeled under the group of functional heartburn, revealed an overall prevalence of $62.00 \%$. Most patients presented with normal manomentric findings (48.39\%). All of the patients presented with negative esophagitis, negative SAP (100\%)/ negative SI $(100 \%)$. (figure 1)

Conclusions Among suspected refractory GERD patients, MII$\mathrm{pH}$ study diagnosed the majority of them with functional heartburn $(62.00 \%)$ followed by persistent acid reflux (20.00\%) and hypersensitive esophagus (18.00\%). Hence, MII$\mathrm{pH}$ monitoring is helpful in the work-up of patients with suspected refractory GERD as it will redirect the course of management. 